\title{
Análise de distribuição de chuva para Santa Maria, RS
}

\author{
Joel C. da Silva ${ }^{1}$, Arno B. Heldwein ${ }^{1}$, Fabrina B. Martins ${ }^{1}$, Gustavo Trentin ${ }^{1}$ \& Edenir L. Grimm
}

\section{RESUMO}

O estudo em pauta teve como objetivo analisar a distribuição da quantidade diária de precipitação e do número de dias com chuva e determinar a variação da probabilidade de ocorrência de precipitação diária, durante os meses do ano, em Santa Maria, RS. Os dados de precipitação utilizados foram obtidos durante 36 anos de observação, na Estação Climatológica do $8^{\circ}$ Distrito de Meteorologia, localizada na Universidade Federal de Santa Maria (29 $43^{\prime} 23^{\prime \prime}$ de latitude Sul e $53^{\circ} 43^{\prime} 15^{\prime \prime}$ de longitude Oeste, altitude $95 \mathrm{~m}$ ). Analisaram-se as seguintes funções de distribuição de probabilidade: gama, Weibull, normal, log-normal e exponencial. As funções que melhor descreveram a distribuição das probabilidades foram gama e Weibull. O maior número de dias com chuva ocorreu durante os meses de inverno porém o volume de precipitação é menor nesses dias, resultando em total mensal semelhante para todos os meses do ano.

Palavras-chave: função de distribuição, precipitação pluvial, gama, Weibull

\section{Analysis of rainfall distribution for Santa Maria, RS, Brazil}

\begin{abstract}
The objectives of this study were to analyze the distribution of total daily rainfall data and the number of rainy-days, and to determine the probability variation of daily precipitation during the months of the year in Santa Maria, Rio Grande do Sul State, Brazil. A 36-year rainfall database measured at the Climatological Station of $8^{\text {th }}$ District of Meteorology, located in Santa Maria Federal University (29 43' $23^{\prime \prime} \mathrm{S}$ and $53^{\circ} 43^{\prime} 15^{\prime \prime} \mathrm{W}$ ) were used in the study. The following probability distribution functions were tested: gamma, Weibull, normal, lognormal and exponential. The functions that best described the frequency distribution were gamma and Weibull. There were more number of rainy days in the winter, but with less amount of rainfall, resulting in similar monthly total precipitation for the twelve months of the year.
\end{abstract}

Key words: distribution function, precipitation, gamma, Weibull 


\section{INTRODUÇÃO}

A determinação prévia da variação dos elementos meteorológicos ao longo do ano, possibilita um planejamento melhor das mais diversas atividades. A precipitação pluvial é um dos elementos meteorológicos de grande importância, pois está diretamente relacionada aos mais diversos setores da sociedade, de forma que o regime pluviométrico afeta a economia, o meio ambiente e a sociedade, como um todo.

Na agricultura, o conhecimento antecipado das condições locais de solo, radiação solar e precipitação pluvial, e sua variação ao longo de um ciclo de cultivo, são significativos para a obtenção de rendimentos satisfatórios, visto que esses fatores são determinantes para o sucesso nos cultivos. Ribeiro \& Lunardi (1997) salientam a importância da caracterização da precipitação em um local para o planejamento de atividades agrícolas, sendo imprescindível também no dimensionamento de reservatórios de água, na elaboração de projetos de proteção e conservação de solos e em atividades de lazer e esportivas.

No estado do Rio Grande do Sul, RS, a precipitação é bem distribuída ao longo do ano porém Berlato (1992) afirma que, embora a precipitação pluvial seja bem distribuída em todas as estações do ano (primavera 26\%, verão $24 \%$, outono $25 \%$ e inverno 25\%), a variabilidade inter-anual desse elemento meteorológico é o principal fator limitante às culturas de primavera-verão, responsável pelas oscilações da produção agrícola.

A maneira de caracterizar a variabilidade da precipitação pluvial é analisar a distribuição dessa variável. Para tanto, são necessários uma análise de distribuição e testes estatísticos para determinar qual função de distribuição de probabilidade é mais adequada para calcular a probabilidade de ocorrer determinado fenômeno, visto que, conforme Cargnelutti Filho et al. (2004), a simples visualização dos dados da amostra de uma variável em um histograma de freqüência é insuficiente para inferir, entre as diversas funções de distribuição de probabilidade conhecidas, a que melhor se ajusta aos dados em estudo.

A função gama tem sido a mais comum no estudo da distribuição dos valores diferentes de zero de precipitação pluvial (Saad, 1990; Assis, 1991; Castro, 1994; Ribeiro \& Lunardi, 1997; Wilks, 1999 e Castellví et al., 2004), porém é possível se utlizar outras funções de distribuição de probabilidade que podem apresentar melhor ajuste que a função gama, pois há variação na distribuição dos valores de precipitação, conforme o período de tempo utilizado para a separação dos dados, segundo o conjunto de dados (médio, total, absoluto, máximo, mínimo) e de acordo com o regime pluviométrico do local em estudo.

Barger \& Thom (1949) sugeriram a distribuição gama como modelo teórico para aproximar as probabilidades de precipitação para períodos mensais ou menores ou, até mesmo, para períodos maiores, em regiões onde é comum a ocorrência de baixos valores de precipitação.

Catalunha et al. (2002), testando as funções de distribuição de probabilidades exponencial, gama, log-normal, normal e Weibull, para descrever a distribuição dos dados de chuva no estado de Minas Gerais, verificaram predominân- cia das funções Weibull, gama e exponencial. Quando foram considerados totais decendiais e totais mensais, a função mais significativa foi a exponencial e nas estimativas para dados diários decendiais e diários mensais a função Weibull apresentou melhor ajuste.

Eltz et al. (1992) utilizaram a função extrema do tipo I, também conhecida como função Gumbel, para determinar o período de retorno de chuvas para a região de Santa Maria, RS, com base em 27 anos de dados registrados na localidade de Boca do Monte. Essa função de distribuição foi escolhida porque se utilizaram dados máximos anuais de intensidade de precipitação. Resultados semelhantes foram obtidos por Damé et al. (1996) para dados de estações meteorológicas instaladas em municípios da região Sul do Rio Grande do Sul, considerando-se valores máximos e mínimos de totais mensais de precipitação.

Além da caracterização da precipitação analisando-se a variável como de distribuição aleatória contínua, é conveniente caracterizar a variabilidade inter-anual do número de dias de chuva, durante certo período. A caracterização do número de dias com chuva pode ser realizada através de funções de distribuição de probabilidade para variáveis aleatórias discretas, considerando-se a ocorrência ou não do evento, ou se utilizando funções de distribuição de probabilidade para variáveis aleatórias contínuas, tendo em vista os totais de dias com chuva em determinado período. A análise do número de dias com chuva através de funções de distribuição de probabilidade pode não ser possível devido à grande variabilidade inter-anual, relacionada aos fenômenos El Niño e La Niña (Fontana \& Almeida, 2002).

Levando-se em conta a importância do tema, os objetivos deste trabalho foram analisar a distribuição do número de dias e da altura diária de precipitação pluvial e determinar a variação da probabilidade de ocorrência de precipitação diária, durante todos os meses do ano para o município de Santa Maria, RS.

\section{MATERIAL E MÉTODOS}

Os dados de precipitação pluvial utilizados para o ajuste das funções de distribuição de probabilidade (fd) foram coletados diariamente, na Estação Climatológica Principal de Santa Maria, instalada no campus da Universidade Federal de Santa Maria (latitude: 29 43’23” S, longitude: $53^{\circ} 43^{\prime} 15^{\prime}$ W $\mathrm{W}$ e altitude: $95 \mathrm{~m}$ ) desde agosto de 1968 até final de julho de 2004, totalizando 36 anos de dados. A coleta dos dados foi realizada todos os dias, às 9 horas, conforme padronização do Instituto Nacional de Meteorologia, ou seja, o volume de água precipitado para dia (n) correspondeu à precipitação ocorrida entre as $9 \mathrm{~h}$ do dia anterior (n-1) e as 9 h do dia (n).

Analisaram-se as funções de distribuição (fd) de probabilidade, gama, Weibull, normal, log-normal e exponencial. Para cada fd foram determinados seus parâmetros, considerando-se os valores de precipitação diária e os valores de precipitação diária transformados através da raiz quadrada e raiz cúbica. 
Todas as distribuições foram selecionadas utilizando-se os testes de Anderson-Darling, Cramér-von Mises, Chi-Quadrado e Kolmogorov-Smirnov (D’Agostino \& Stephens, 1986, Assis, 1991, Catalunha et al., 2002). Para considerar todos os testes citados e resultar em uma escolha melhor das fd, criou-se um sistema de classificação.

O critério inicial do sistema de classificação consiste em selecionar as fd em que o valor de probabilidade indicado por pelo menos um dos testes citados, foi maior que o nível de significância de $10 \%$, descartando-se as demais; em seguida, as fd foram classificadas de acordo com o resultado dos testes do Chi-Quadrado $\left(\chi^{2}\right)$ e de Kolmogorov-Smirnov (KS), sendo enumeradas a partir do maior valor de probabilidade (p), mais significativas, para o menor valor. A partir dessas duas classificações criou-se uma terceira classificação, que considerava a soma das duas classificações, do teste de $\chi^{2}$ e de KS, que também foi enumerada em ordem crescente, resultando em uma classificação geral de todas as fd selecionadas. Essa última classificação, dita "geral”, foi necessária pois se observou, em alguns casos, que o resultado do teste do $\chi^{2}$ indicava determinada fd como sendo mais significativa, porém o teste de KS indicava outra fd.

As duas fd que foram mais bem classificadas, pela classificação geral, foram selecionadas, permanecendo como critério principal na identificação da melhor fd os resultados do teste de $\chi^{2}$, de vez que, conforme Catalunha et al. (2002) o teste de $\chi^{2}$ é considerado superior ao teste de KS para análises desta natureza.

Após o ajuste das fd foi possível calcular as probabilidades de um valor de precipitação diária ser maior que $\mathrm{x}\left[\mathrm{P}_{\mathrm{m}}(\mathrm{X}>\mathrm{x})\right]$, durante os meses $(\mathrm{m})$, através de:

$$
\mathrm{P}_{\mathrm{m}}(\mathrm{X}>\mathrm{x})=\left[1-\mathrm{P}_{\mathrm{m}}(\mathrm{x})\right] \mathrm{F}(\mathrm{x})
$$

em que $\mathrm{P}_{\mathrm{m}}(\mathrm{x})$ é a probabilidade de ocorrer um valor de precipitação diária menor ou igual a $\mathrm{x}$, calculada pela função densidade de probabilidade cumulativa da fd, determinada para cada mês $m$ e $F(x)$ é a freqüência de ocorrência de precipitação durante o mês $m$.

A freqüência de ocorrência representa o número de dias nos quais ocorreu precipitação, em cada mês, para todos os anos da série de dados meteorológicos disponível, em relação ao total de dias analisados.

As soluções para as funções testadas podem ser encontradas em Catalunha et al. (2002), exceto que foi utilizada a função Weibull com três parâmetros, reduzidos para dois, pois a estimativa desta função foi realizada considerando-se o parâmetro de posição igual a zero.

Para os meses em que não foi possível estimar nenhuma fd, utilizando-se todos os dados de altura de chuva, dividiram-se os dados em conjuntos menores. Quando não foi possível calcular a probabilidade para determinados valores, a probabilidade foi determinada utilizando-se a freqüência observada, ao invés de $\mathrm{P}_{\mathrm{m}}(\mathrm{x})$.

Para analisar a distribuição do número de dias com chuva durante os meses do ano, realizou-se o mesmo procedimento descrito para a altura de chuva, utilizando-se o nú- mero de dias com chuva durante cada mês do ano, para toda a série de dados disponível.

\section{RESULTADOS E DISCUSSÃO}

Através da análise de distribuição de freqüência foi possível ajustar funções de distribuição de freqüência (fd) para os dados diários de precipitação pluvial, separados por mês, para oito meses do ano, exceto os meses de junho, julho, agosto e novembro (Tabela 1 ).

Tabela 1. Parâmetros escalar e de forma, ajustados às funções de distribuição de probabilidades (fd), Weibull (W) e Gama (G), para cada mês, utilizando-se as variáveis (Var) precipitação diária (Prec) ou raiz quadrada de precipitação $\left(\operatorname{Precr}^{1 / 2}\right)$, freqüência de ocorrência das variáveis ( $\mathrm{F} \%$ ) e probabilidade para o teste do $\chi^{2}$

\begin{tabular}{ccccrcc}
\hline Mês & Var & fd & F (\%) & $\begin{array}{r}\text { Parâmetro } \\
\text { Escalar }\end{array}$ & $\begin{array}{c}\text { Parâmetro } \\
\text { de Forma }\end{array}$ & $\begin{array}{c}\text { Prob } \\
\chi^{2}\end{array}$ \\
\hline Jan & Precr $^{1 / 2}$ & W & 36,1 & 3,4539 & 1,5039 & 0,5615 \\
\hline Jan & Prec & W & 36,1 & 11,9295 & 0,7520 & 0,5264 \\
\hline Fev & Prec & W & 40,0 & 10,5856 & 0,7493 & 0,4423 \\
\hline Fev & Prec & G & 40,0 & 19,3930 & 0,6490 & 0,3409 \\
\hline Mar & Prec & G & 32,7 & 26,1461 & 0,5589 & 0,4213 \\
\hline Mar & Prec & W & 32,7 & 11,1931 & 0,6740 & 0,2172 \\
\hline Abr & Prec & W & 36,9 & 9,1027 & 0,6011 & 0,8011 \\
\hline Abr & Prec & G & 36,9 & 28,5821 & 0,4759 & 0,1427 \\
\hline Mai & Prec & W & 35,0 & 8,2269 & 0,5800 & 0,8858 \\
\hline Mai & Prec & G & 35,0 & 28,3384 & 0,4534 & 0,6942 \\
\hline Jun* & Prec & G & 28,43 & 27,0449 & 0,6654 & 0,1421 \\
\hline Jul* & Prec & G & 29,69 & 21,2849 & 0,7454 & 0,2184 \\
\hline Jul* & Prec & W & 29,69 & 14,5062 & 0,8353 & 0,1294 \\
\hline Ago* & Precr ${ }^{1 / 2}$ & W & 24,78 & 2,61061 & 1,1462 & 0,2025 \\
\hline Ago* & Prec & W & 24,78 & 13,0994 & 0,7712 & 0,1568 \\
\hline Set & Prec & G & 36,1 & 23,7513 & 0,5832 & 0,3813 \\
\hline Set & Prec & W & 36,1 & 10,9680 & 0,6959 & 0,2283 \\
\hline Out $^{*}$ Prec & W & 35,5 & 11,4269 & 0,7106 & 0,4138 \\
\hline Out & Prec & G & 35,5 & 23,6219 & 0,6012 & 0,1795 \\
\hline Nov* & Prec & G & 25,5 & 22,5195 & 0,7095 & 0,4732 \\
\hline Nov* & Prec & W & 25,5 & 14,3224 & 0,8082 & 0,3398 \\
\hline Dez & Prec & W & 30,8 & 11,6427 & 0,6792 & 0,5334 \\
\hline Dez & Prec & G & 30,8 & 26,8003 & 0,5658 & 0,3977 \\
\hline
\end{tabular}

* Para esses meses foram utilizados dados de precipitação maiores ou iguais a um, para que houvesse ajuste de pelo menos uma fd

Comparando-se as fd através do valor de probabilidade do teste do $\chi^{2}$, constata-se que as duas melhores fd foram Weibull e gama. A função Weibull foi superior, de acordo com os critérios utilizados, para a maioria dos meses (Tabela 1), exceto para os meses de março, junho, julho, setembro e novembro, nos quais a fd que melhor se ajustou foi a gama. Nos meses de junho, julho e novembro, foram usados dados maiores ou iguais a um.

As transformações dos dados, para raiz quadrada e para raiz cúbica, não melhoraram o ajuste das fd, exceto nos meses de janeiro e agosto, em que ocorreu uma pequena diferença, demonstrada no valor de $\mathrm{p}$ do teste de $\chi^{2}$ (Tabela 1 ).

Utilizando-se os parâmetros das fd ajustadas calculou-se a probabilidade de ocorrer precipitação maior ou menor que 
determinado valor, em um dia, no período de dezembro a maio, setembro e outubro, utilizando-se a Eq. 1. Desta maneira, foi necessário o conhecimento dos respectivos parâmetros das funções de densidade de probabilidade, gama e Weibull, para cada mês e da freqüência de ocorrência de precipitação durante cada mês (Tabela 1). As fd e sua descrição podem ser obtidas em Catalunha et al. (2002). Os dados apresentados na Tabela 1 são semelhantes aos obtidos por Catalunha et al. (2002), que também constataram superioridade da função Weibull em relação às demais, para valores diários mensais observados durante o período chuvoso, em Minas Gerais.

Não foi possível ajustar nenhuma fd, utilizando-se todos os dados de precipitação, para os meses de junho, julho, agosto e novembro, razão por que se procedeu à análise e determinação das fd, considerando-se apenas valores de precipitação maiores que um, para esses meses. Esta subdivisão dos dados tornou possível o ajuste de pelo menos uma fd, para os meses citados (Tabela 1). Para esses meses, o cálculo da probabilidade (Eq. 1) deve ser realizado considerando-se os valores de $\mathrm{F}(\%)$ (Tabela 1$)$.

Constatou-se que, durante os meses de junho, julho e agosto, as precipitações são mais freqüentes e em menor quantidade. Mais que $50 \%$ das chuvas ocorridas nesses meses têm altura menor que $5 \mathrm{~mm}$, com a ocorrência de alguns valores com precipitação maior que $50 \mathrm{~mm}$, em aproximadamente $5 \%$ dos casos. Essa é a principal razão pela qual não foi possível o ajuste satisfatório de alguma fd para os dados de precipitação diária ocorrida durante esses meses.

Para o mês de novembro, a freqüência de ocorrência de precipitação com volume menor que $5 \mathrm{~mm}$ foi em torno de $44 \%$. Observou-se aumento na freqüência de valores de precipitação, entre 30 e 55 mm (10\%), contrariando a tendência de diminuição da freqüência com o aumento da precipitação. A inexistência de ajuste de alguma fd, para este mês, se deveu à característica "bi-modal” da distribuição dos dados diários de chuva.

Analisando-se a variação de probabilidade de ocorrência de precipitação durante um dia, para cada mês, apresentada na Figura 1, verifica-se que a probabilidade é variável ao longo do ano e mais acentuada para a probabilidade de ocorrer qualquer valor de precipitação (P0) e de ocorrer precipitação maior que $1 \mathrm{~mm}$ (P1), pois nos dois meses mais frios ocorrem precipitações menores e mais freqüentes que nos demais meses. Aproximadamente $70 \%$ dos valores da precipitação em todos os meses estão entre zero e $1 \mathrm{~mm}$ e ocorre uma probabilidade de aproximadamente 30\% de haver precipitação durante 1 dia, maior que $1 \mathrm{~mm}$ (Figura 1) e de apenas $15 \%$ para $\mathrm{P}$ maior que $10 \mathrm{~mm}$ para qualquer mês do ano. Para precipitações superiores a $50 \mathrm{~mm}$ a probabilidade é de aproximadamente $1,4 \%$ para todos os meses do ano (Figura 1).

Apesar de se observar diferenças entre as fd, obtidas pelo teste do $\chi^{2}$ (Tabela 1 ), foi pouca a diferença entre os valores de probabilidades estimadas pelas duas funções, sendo perceptível apenas para os valores maiores que $10 \mathrm{~mm}$ (P10) em que, geralmente, a função gama estima maiores probabili-

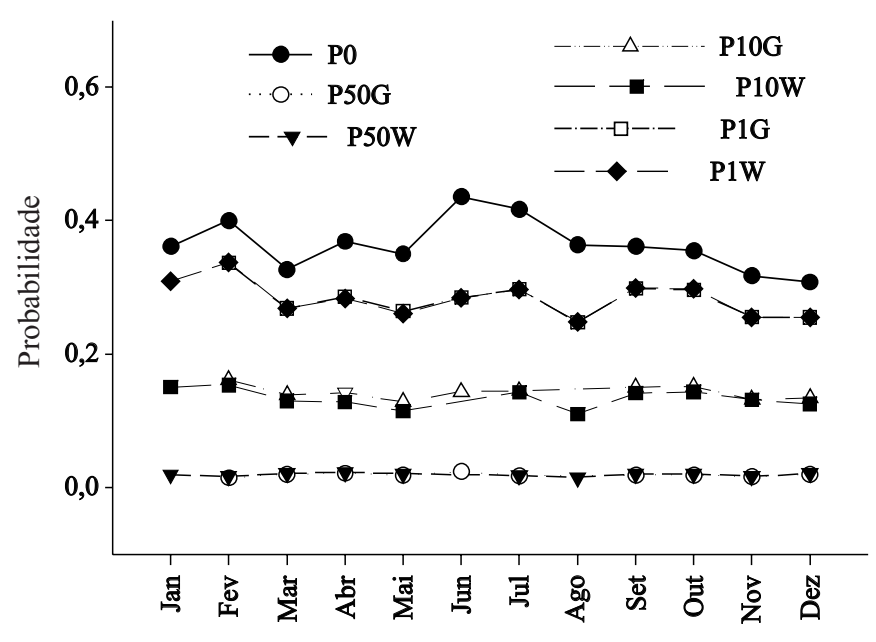

Figura 1. Probabilidades de ocorrência de precipitação maior que zero (P0), maior que $1 \mathrm{~mm}$ (P1), maior que $10 \mathrm{~mm}$ (P10) e maior que $50 \mathrm{~mm}$ (P50), estimadas pelas funções gama $(G)$ e Weibull (W) para o município de Santa Maria, RS

dades que a função Weibull (Figura 1). Na Figura 2, observando-se os coeficientes da equação linear para linha de tendência estimada para a relação de probabilidades estimadas com as duas fd, verifica-se que o coeficiente angular da reta se aproximou de 1 e o coeficiente linear se aproximou de zero, demonstrando que qualquer uma das funções pode ser utilizada para a estimativa da probabilidade de ocorrer qualquer valor de precipitação durante um dia. A tendência da função gama superestimar as probabilidades em relação à Weibull é de apenas 2\% (Figura 3).

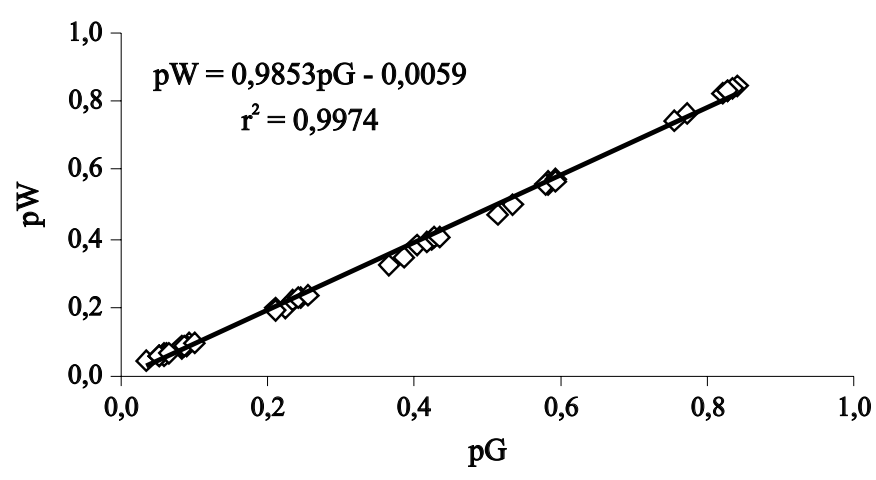

Figura 2. Relação entre as probabilidades de ocorrência de precipitação estimadas pelas funções Weibull (pW) e gama (pG) para o município de Santa Maria, RS

A Figura 3 apresenta a distribuição da ocorrência do número de dias com chuva para Santa Maria. Constatou-se no período de junho a agosto, a ocorrência de um número maior de dias com precipitação, associado a uma atuação maior das frentes frias, conforme Grimm (2003). A predominância do maior número de dias com chuva no inverno decorre da grande atividade da circulação secundária do ar, sendo acentuado o domínio das altas pressões nessa época do ano, produzindo chuvas mais duradouras especialmente quando acompanhadas dos ventos de sul, marítimos e, por isso mesmo, carregados de umidade (Machado, 1950). 

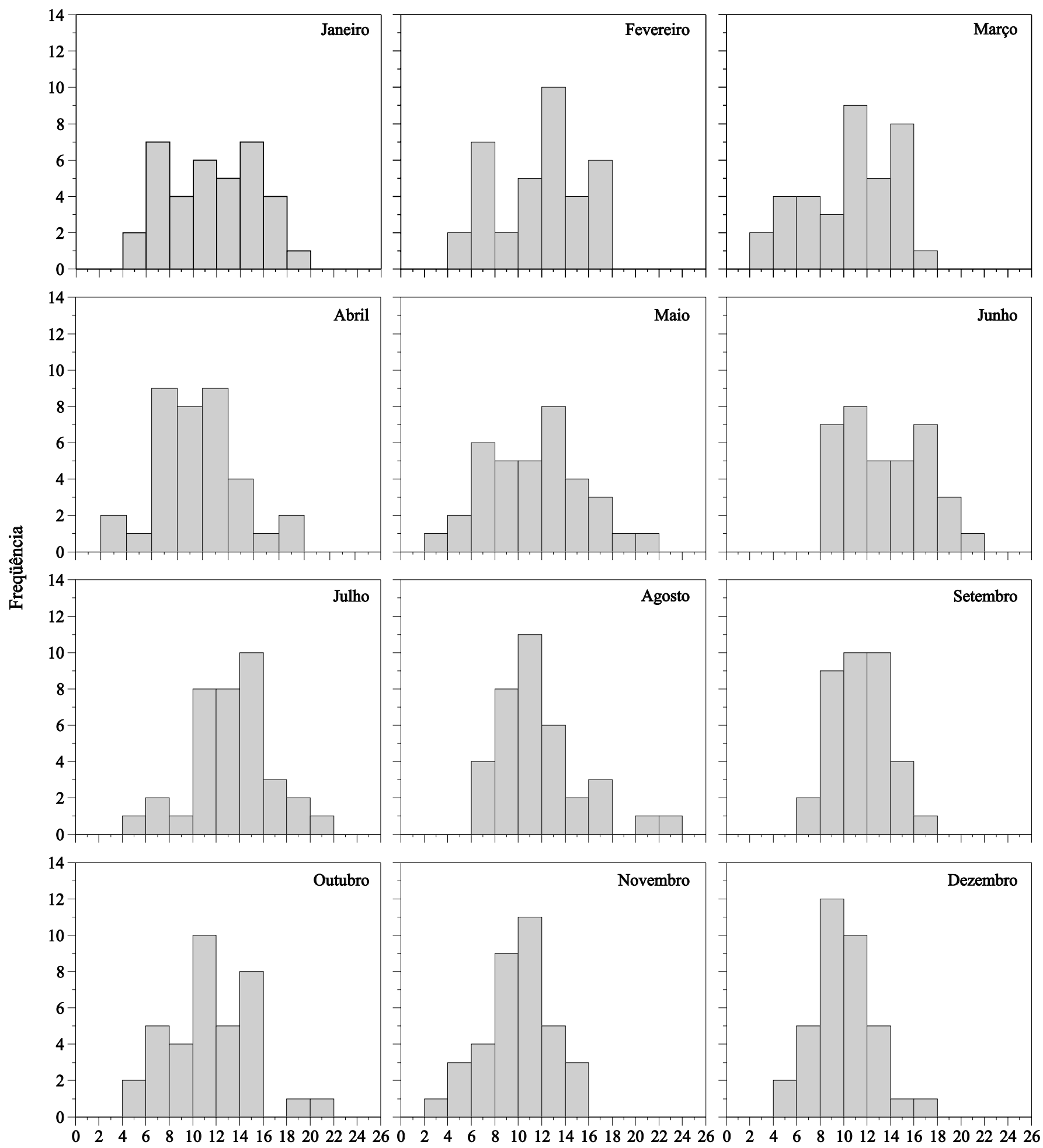

Número de dias

Figura 3. Distribuição da freqüência do número de dias com chuva no município de Santa Maria, RS, no período de agosto de 1968 a julho de 2004

O maior número de dias com precipitação observado em um mês da série histórica analisada foi de 22 dias, em agosto de 1975; afora este valor, registraram-se, ainda, 21 dias nos meses de maio e agosto de 1983 e 20 dias nos meses junho de 1997, agosto de 1972 e outubro de 2002. O menor número de dias com precipitação observado em um mês foi de 2 dias, no mês de março de 1982. Ainda se observaram apenas 3 dias com precipitação nos meses de março de 1981, maio de 1978 e novembro de 1985. O número de dias com precipitação pluvial foi semelhante nas quatro estações do ano, resultado similar ao encontrado por Fontana \& Almeida (2002) utilizando dados mensais para o estado do Rio Grande do Sul. 


\section{CONCLUSÕES}

1. As funções de distribuição de probabilidades que melhor se ajustaram aos dados diários de precipitação, foram gama e Weibull, não havendo diferença entre as probabilidades calculadas por essas funções.

2. A probabilidade de ocorrer diferentes alturas diárias de precipitação é variável ao longo do ano. A distribuição do número de dias com chuva também é variável nos meses do ano.

3. Nos meses de junho e julho a chuva é mais freqüente mas o total mensal é semelhante aos demais meses do ano.

\section{AGRADECIMENTOS}

Os autores agradecem à CAPES e ao CNPq pelas bolsas de Doutorado e Mestrado e de produtividade recebidas. Os autores também agradecem ao comitê editorial e aos revisores da Revista Brasileira de Engenharia Agrícola e Ambiental pela colaboração à qualidade deste artigo.

\section{LITERATURA CITADA}

Assis, F. N. de. Modelagem da ocorrência e da quantidade de chuva e de dias secos em Piracicaba, SP, e Pelotas, RS. Piracicaba: ESALQ, 1991. 134p. Tese Doutorado

Barger, G. L.; Thom, H. C. S. Evaluation of drought hazard. Agronomy Journal, Madison, v.41, n.11, p.519-526, 1949.

Berlato, M. A. As condições de precipitação pluvial no Estado do Rio Grande do Sul e os impactos da estiagem na produção agrícola. In: Bergamaschi, H. (coord.) Agrometeorologia aplicada à irrigação. Porto Alegre: UFRGS, 1992. p.11-23.

Cargnelutti Filho, A.; Matzenauer, R.; Trindade, J. K. da. Ajustes de funções de distribuição de probabilidade à radiação solar global no Estado do Rio Grande do Sul. Pesquisa Agropecuária Brasileira, Brasília, v.39, n.12, p.1157-1166, 2004.
Castellví, F.; Mormeneo, I.; Perez, P. J. Generation of daily amounts of precipitation from standard climatic data: a case study for Argentina. Journal of Hydrology, Amsterdam, v.289, n.1-4, p.286-302, 2004.

Castro, R. Distribuição probabilística da freqüência de precipitação na região de Botucatu, SP. Botucatu: UNESP, 1994. 101p. Dissertação Mestrado

Catalunha, M. J.; Sediyama, G. C.; Leal, B. G.; Soares, C. P. B.; Ribeiro, A. Aplicação de cinco funções densidade de probabilidade a séries de precipitação pluvial no Estado de Minas Gerais. Revista Brasileira de Agrometeorologia, Santa Maria, v.10, n.1, p.153-162, 2002.

D’Agostino, R. B., Stephens, M. A. Goodness-of-fit techniques. New York: Marcel Dekker, Inc., 1986. 286p.

Damé, R. C. F.; Teixeira, C. F. A.; Souto, M. V. Análise de freqüência dos dados de precipitação pluvial de algumas estações agroclimatológicas da região Sul do Rio Grande do Sul. Ciência Rural, Santa Maria, v.26, n.3, p.351-355, 1996.

Eltz, F. L. F.; Reichert, J. M.; Cassol, E. A. Período de retorno de chuvas em Santa Maria, RS. Revista Brasileira de Ciência do Solo, Campinas, v.16, n.2, p.265-269, 1992.

Fontana, D. C. ; Almeida, T. S. Climatologia do número de dias com precipitação pluvial no estado do Rio Grande do Sul. Revista Brasileira de Agrometeorologia, Santa Maria, v.10, n.2, p.341-349, 2002.

Grimm, A. M. The El Niño impact on the summer monsoon in Brazil: regional processes versus remote influences. Journal of Climate, Boston, v.16, n.3, p.263-280, 2003.

Machado, F. P. Contribuição ao estudo do clima do Rio Grande do Sul. Rio de Janeiro: IBGE/Conselho Nacional de Geografia, 1950. 91p.

Ribeiro, A. M.; Lunardi, D. M. C. A precipitação mensal provável para Londrina - PR, através da função gama. Energia na Agricultura, Botucatu, v.12, n.4, p.37-44, 1997.

Saad, J. C. C. Estudo das distribuições de freqüência da evapotranspiração de referência e da precipitação pluvial para fins de dimensionamento de sistemas de irrigação. Piracicaba: ESALQ/USP, 1990. 124p. Dissertação Mestrado

Wilks, D. S. Interannual variability and extreme-value characteristics of several stochastic daily precipitation models. Agricultural and Forest Meteorology, Amsterdam, v.93, n.3, p.153-169, 1999. 